\title{
Substitutions of rtL228 and/or L229 are involved in the regulation of replication and HBsAg secretion in hepatitis B virus, and do not affect susceptibility to nucleos(t)ide analogs
}

\author{
BO QIN ${ }^{1,2}$, YECHAO ZHOU ${ }^{1}$, GUOZHONG ZHOU ${ }^{1}$, XIUPING XU $^{1}$, YANAN WANG ${ }^{1}$ and JINKUN CHEN ${ }^{2}$ \\ ${ }^{1}$ Department of Clinical Laboratory Center, Shaoxing People's Hospital, Shaoxing Hospital of Zhejiang University; \\ ${ }^{2}$ Shaoxing Center for Disease Control and Prevention, Shaoxing, Zhejiang 312000, P.R. China
}

Received December 27, 2016; Accepted August 22, 2017

DOI: $10.3892 / \mathrm{mmr} .2017 .7778$

\begin{abstract}
Nucleos(t)ide analogs (NAs) are widely used in the treatment of hepatitis B virus (HBV) and human immunodeficiency virus (HIV). The mutation L210W of HIV-1 reverse transcriptase (RT) is one of the six principal mutations which confer in vivo resistance to zidovudine. Due to the similar 3D-structure and high conservation between HIV-RT and HBV-RT, the present study aimed to clarify whether corresponding mutations in HBV may decrease its susceptibility to relevant NAs. Mutations including rtL228C/W, rtL229W and $\mathrm{rtL} 228 \mathrm{~W} / \mathrm{L} 229 \mathrm{~W}$ were introduced into a HBV replication competent plasmid by fusion polymerase chain reaction. Replication capacity, HBs/e antigen (Ag) levels and susceptibility to NAs were subsequently analyzed in vitro. Single or combination mutations of rtL228 and rtL229 impaired HBV replication. Decreased HBsAg secretion in the supernatant and production in the cell lysate wasobserved with single rtL229W or in combination with rtL228W, while there was no significant difference between wild-type and mutant $\mathrm{HBV}$ with regard to the level of $\mathrm{HBeAg}$ in the supernatant and susceptibility to commonly-used NAs. Substitution mutations of rtL228 and/or L229 in HBV did not alter the susceptibility of the virus to NAs, although replication and HBsAg secretion were affected.
\end{abstract}

\section{Introduction}

Persistent infection with hepatitis B virus (HBV) is a leading cause of acute and chronic necroinflammatory liver diseases, hepatic cirrhosis and hepatocellular carcinoma (HCC) (1).

Correspondence to: Professor Bo Qin, Department of Clinical Laboratory Center, Shaoxing People's Hospital, Shaoxing Hospital of Zhejiang University, 568 Zhongxing North Road, Shaoxing, Zhejiang 312000, P.R. China

E-mail: qinbo0809@hotmail.com

Key words: hepatitis B virus, quasispecies, nucleos(t)ide analogs, susceptibility, resistance mutation
HBV represents a major health issue worldwide, $>360$ million chronically-infected patients at risk of developing liver cirrhosis or HCC $(1,2)$. HBV is an hepadnavirus which replicates via reverse transcription of pregenomic RNA (pgRNA), sharing a similar life cycle with human immunodeficiency virus (HIV) (3). Nucleos(t)ide analogs (NAs), extensively used to inhibit reverse transcription in HBV and/or HIV, comprise lamivudine (LMV), adefovirdipivoxil (ADV), entecavir (ETV), telbivudine (LdT), tenofovir (TDF), zidovudine (AZT), stavudine (D4T) and dideoxyinosine (DDI) (4-6).

In patients, HBV and/or HIV exists in the form of quasispecies due to the error-prone reverse transcriptase (RT), which is responsible for the variety of drug-resistant mutants (7). In vivo and in vitro, NAs mimic physiological nucleosides in terms of uptake and metabolism and are incorporated into newly-synthesized DNA chains, leading to synthesis inhibition via the termination of DNA replication (8). The replication of drug-resistant HBV/HIV variants during multidrug therapy is considered to be a primary cause of treatment failure (9). Under antiviral pressure, variants may contribute to the production and further selection of replication-competent resistance mutants, which will spread to other cells and may eventually replace the wild-type virus (10). Long-term antiviral therapy is therefore required, which frequently leads to the emergence and selection of drug-resistant mutations in the HIV and HBV viral polymerase $(9,11)$.

NA-resistant mutations inhibit the anti-HBV effect and induce virological advancement and hepatopathological progression (6). A number of mutations have been reported to account for drug resistance, as reviewed below. Mutations of rtM204I/V in the YMDD motif of HBV-RT lead to LMV resistance (LMVr) and LdT resistance (LdTr). ETV resistance (ETVr) has additionally been observed in LMVr patients (6). With rt M204I/V mutation, a combination of mutations in the $\mathrm{B}, \mathrm{C}$ or D domains of RT may lead to ETVr, including rtI169T, rtL180M, rtS184G, rtA186T, rtS202I, rtM204V, rtM250V (6). The substitution mutations rtA181V/T, rtN236T, rtN238R, rtT240Y and rtN248H are able to reduce the anti-HBV effects of ADV (12), while rtP177G and rtF249A have been reported to induce TDF resistance; rtV214A, rtQ215S and rtA194T required further confirmation (9). Owing to the decreased replicative capacity of resistance mutants, for survival, 
compensatory mutations of $\mathrm{HBV}$ which restore viral replication are selected for, including rtL80V/I, rtI163V, rtI169T, rtV173L, rtT184S/G, rtS202I, rtQ215S and rtQ267H (13).

As a commonly-used anti-HBV reagent, NAs were originally used for the treatment of HIV as antiretroviral drugs (9). Due to their similar replication processes, involving the reverse transcription of pgRNA, HIV is analogous to HBV, and resistance mutations occur following long-term anti-HIV therapy with NAs (9). The majority of resistance mutations are located within the palm and finger subdomains of HIV-1-RT. The combination of M41L, L210W, and T215Y decreases TDF susceptibility by $\sim 4$-fold (14). Mutation L210W has been frequently detected in viruses sequenced from patients undergoing AZT therapy (15).

In a previous study, given the homology between HIV-RT and HBV polymerase, their primary amino acids sequences were aligned, and corresponding positions were identified and mapped (9). Based on this model, the distances of a given amino acid (aa) residue in the HBV-RT to an NA substrate were able to be estimated. Notably, positions 210-211 of HIV-RT and 228-229 of HBV-RT are conserved double leucine residues. As summarized above, L210W in HIV-RT is one of the six principal mutations that confer in vivo resistance to certain NAs, particularly AZT, which appears following T215Y/F $(14,16)$. Crystallographic studies suggested that the aromatic side chain of Trp210 was able to stabilize the interaction between Phe/Tyr215 and the dNTP-binding pocket (14). In present study, the L228 and L229 mutations were investigated. A series of HBV constructs harboring different mutations were constructed. The replication capacity, antigen expression level and resistance phenotype were analyzed in vitro. The results demonstrated that rtL228 and/or L229 of HBV-RT did not affect susceptibility to NAs, although replication and HBs/eAg secretion were altered.

\section{Materials and methods}

Cells. Hepatoma cell lines (Huh7 and HepG2), which were kindly provided by Professor Xinwen Chen (Wuhan Institute of Virology, Chinese Academy of Sciences, Wuhan, China), were cultured in Dulbecco's modified Eagle's medium (Invitrogen; Thermo Fisher Scientific, Inc., Waltham, MA, USA) supplemented with $10 \%$ fetal bovine serum (Gibco; Thermo Fisher Scientific, Inc.), 2 mM/l glutamine, $100 \mathrm{IU} / \mathrm{ml}$ penicillin and $100 \mathrm{IU} / \mathrm{ml}$ streptomycin at $37^{\circ} \mathrm{C}$ in a $5 \% \mathrm{CO}_{2}$ atmosphere incubator (17).

NAs. A total of eight types of NA were used in the present study: 3TC (GlaxoSmithKline, plc, Brentford, UK), ADV (Gilead Sciences, Inc., Foster City, CA, USA), ETV (Bristol-Myers Squibb Co., New York, NY, USA), LdT (Novartis International AG, Basel, Switzerland), TDF (Gilead Sciences, Inc.), AZT (Teva Canada, Ltd., Whitchurch-Stouffville, ON, Canada), D4T (Bristol-Myers Squibb Co.) and DDI (Teva Canada, Ltd.). The NAs were dissolved in the appropriate solution according to the manufacturer's protocol, and were used in the assays at the indicated concentrations $(9,18)$.

Sequences and plasmid constructs. HIV-RT (GenBank accession no. HQ718313; https://www.ncbi.nlm.nih. gov/nuccore/HQ718313) and HBV polymerases (GenBank
Table I. Primers for the construction of MT HBV plasmids and RT-qPCR.

\begin{tabular}{ll}
\hline Name & \multicolumn{1}{c}{ Sequence (5'-3') } \\
\hline p-RT-mt-F & TCTTCTCGAGGATTGGGGACC \\
p-RT-mt-R & GCAGCCATGGAAACGATGTAT \\
p-rtL228C-F & ATTTTTGTTTGTCTTTGGGTATACAT \\
p-rtL228C-R & ATGTATACCCAAAGACAAACAAAAAT \\
p-rtL228W-F & ATTTTTGGTTGTCTTTGGGTATACAT \\
p-rtL228W-R & ATGTATACCCAAAGACAACCAAAAAT \\
p-rtL229W-F & ATTTTCTTTGGTCTTTGGGTATACAT \\
p-rtL229W-R & ATGTATACCCAAAGACCAAAGAAAAT \\
p-rtL228W/ & ATTTTTGGTGGTCTTTGGGTATACAT \\
L229W-F & \\
p-rtL228W/ & ATGTATACCCAAAGACCACCAAAAAT \\
L229W-R & \\
p-HBV-rc-F & GTTGCCCGTTTGTCCTCTAATTC \\
p-HBV-rc-R & GGAGGGATACATAGAGGTTCCTT \\
\hline
\end{tabular}

Underlining, restriction enzyme cleavage sites for fusion PCR. Bold, substitution mutations for MT pHBV1.3 construction. MT, mutant; F, forward; R, reverse; HBV, hepatitis B virus; RT-qPCR, reverse transcription-quantitative polymerase chain reaction.

accession no. CAA48354.1; https://www.ncbi.nlm.nih. gov/protein/CAA48354.1), were used for their primary amino acids sequences alignment. For the construction of the replication-competent plasmid with aa substitutions, the pHBV1.3 plasmid (1.3-fold over-length HBV genome; subtype, ayw) was used as a backbone $(9,13)$. Mutations were introduced into wild-type (WT) HBV via polymerase chain reaction (PCR)-based mutagenesis using the primer pairs P-RT-mt-F, P-RT-mt-R and primers with specific mutations listed in Table I, as described previously (9). Plasmids containing mutation sites were identified by sequencing. Serial plasmids pHBV1.3-rtX (X, mutations including rtL228W) were producedusing DH5 $\alpha$ Escherichia coli and extracted using a Plasmid Plus Midi kit (Qiagen GmbH, Hilden, Germany) (13).

ELISA analysis. HBsAg in the supernatant and the cell lysate, $\mathrm{HBeAg}$ in the supernatant from indicated HBV-bearing plasmid-transfected Huh7/HepG2 cells were analyzed using a diagnostic kit for HBsAg (cat. no. 201606212) and HBeAg (cat. no. 201606122) detection (Shanghai Kehua Bio-Engineering Co., Ltd., Shanghai, China) according to the manufacturer's protocol (17).

Detection of virion-associated HBV DNA by southern blotting and quantitative (q)PCR analysis. Huh7 or HepG2 cells $\left(1-2 \times 10^{6}\right)$ were transfected with $4 \mu \mathrm{g}$ replication-competent plasmid, with or without the indicated concentration of the different NAs, using Lipofectamine 2000 (Invitrogen; Thermo Fisher Scientific, Inc.). HBV-encapsid at edreplicative intermediates were collected and purified from intracellular core particles, according to the method described in a previous study (9). Cells were washed using ice-cold PBS and lysed with $400 \mu \mathrm{l}$ lysis buffer at $4^{\circ} \mathrm{C}$ for $15 \mathrm{~min}$. The nuclei were 
A

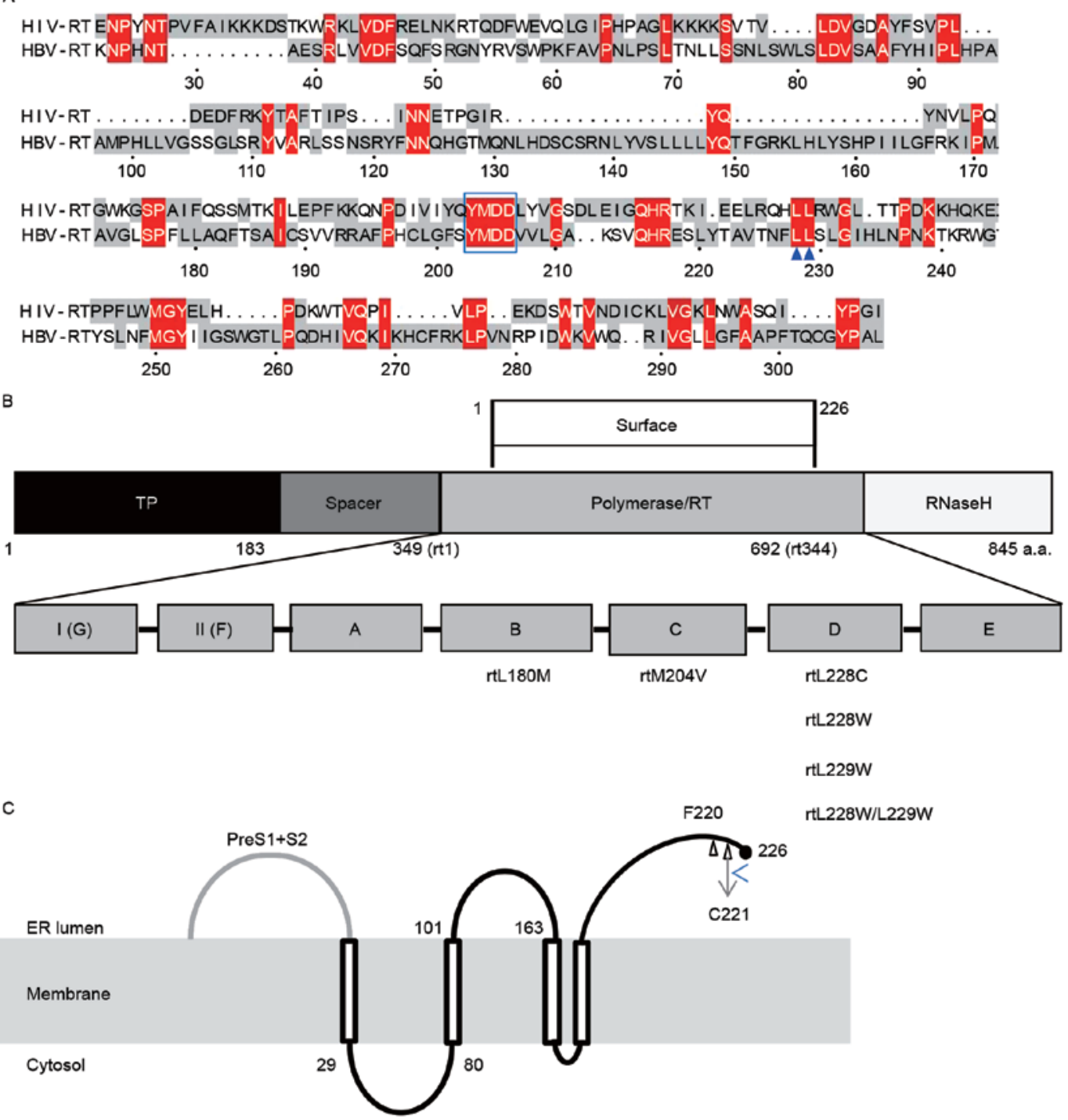

Figure 1. Sequence alignment and structure of HBV RT. (A) Amino acid sequence alignment of HBV-RT and HIV-RT. Colored regions indicate areas of sequence conservation. Amino acids in red were predicted to be potential regions which may interact with NAs. Within these, L228 and L229, which are highly conservative with the corresponding positions in HIV, are marked by arrowheads. (B) Structure of HBV RT and location of target mutations. HBV polymerase is divided into four regions: TP, spacer, RT and RNaseH. RT, containing the whole surface protein, is subdivided into seven regions, A-G. All the amino acid substitutions examined in the present study are located in region D. (C) Proposed topology of HBV small envelope protein. The small envelope protein likely traverses the ER membrane four times: TMD-I, between residues 4 and 28; TMD-II, between 100 and 164; TMD-III, between173 and193; TMD-IV, between 202 and 222; and the C terminal, between residues 223 and 226, where sF220 and sC221G are located. HBV, hepatitis B virus; HIV, human immunodeficiency virus; NA, nucleos(t)ide analogs; RT, reverse transcriptase; ER, endoplasmic reticulum; TMD, transmembrane domain; TP, terminal protein.

pelleted by centrifugation, and the supernatant was adjusted to $10 \mathrm{mM} \mathrm{MgCl}$ and treated with $100 \mathrm{mg} / \mathrm{ml}$ DNase I (Roche Diagnostics $\mathrm{GmbH}$, Mannheim, Germany) at $37^{\circ} \mathrm{C}$ for $30 \mathrm{~min}$ to digest the input DNA (transfected plasmid), which was terminated with a final concentration of $25 \mathrm{mM}$ EDTA. A total of $0.5 \mathrm{mg} / \mathrm{ml}$ proteinase $\mathrm{K}$ (Qiagen $\mathrm{GmbH})$ and $1 \%$ SDS was used to digest the excess proteins in the supernatant at $56^{\circ} \mathrm{C}$ for $2 \mathrm{~h}$. HBV DNA was extracted using phenol/chloroform followed by isopropanol precipitation, resuspended in TE buffer, and subjected to agarose gel electrophoresis, followed by denaturation and Southern blotting with a ${ }^{32} \mathrm{P}$-labeled full length HBV DNA probe. Hybridization signals were acquired and analyzed using Phosphor Imager (Cyclon; Packard Instrument Company, Inc., Meriden, CT, USA) (13).

HBV DNA was additionally quantified by qPCR analysis using primers [relaxed circular (RC)-sense and RC-antisense;
Table I] specific to HBV RC genomes in a SYBR-Green reaction mix (Roche Diagnostics GmbH). Plasmid pBSK-HBV1.3-WT was quantified and 10-fold diluted as a standard curve (13). All samples were analyzed in triplicate, and every value is presented as the mean of at least three independent experiments.

Statistical analysis. In the present study, the statistical analysis was performed using GraphPad (GraphPad Software, Inc., La Jolla, CA, USA). Each value is presented as the mean of three independent experiments. Differences in pairwise comparisons were determined using a Student's t-test for statistical significance. Differences in multiple comparisons were determined using a one-way analysis of variance followed by the Least Significant Difference post hoc test. $\mathrm{P}<0.05$ was considered to indicate a statistically significant difference. Results are presented as the means \pm standard deviation. 
Table II. List of co-mutations between surface antigens and HBV polymerase.

Mutations of HBV-RT

HBsAg corresponding mutations

\begin{tabular}{ll}
\hline $\mathrm{rtL} 228 \mathrm{C}$ & $\mathrm{sF} 220 \mathrm{~V}$ \\
$\mathrm{rtL} 228 \mathrm{~W}$ & $\mathrm{sF} 220 \mathrm{G}$ \\
$\mathrm{rtL} 229 \mathrm{~W}$ & $\mathrm{sC} 221 \mathrm{G}$ \\
$\mathrm{rtL} 228 \mathrm{~W} / \mathrm{L} 229 \mathrm{~W}$ & $\mathrm{sF} 220 \mathrm{G} / \mathrm{sC} 221 \mathrm{G}$
\end{tabular}

Overlap between the surface protein and polymerase genes leads to single nucleotide substitutions causing alterations to the surface protein and polymerase. HBV, hepatitis B virus; RT, reverse transcriptase; Ag, antigen.

\section{Results}

Sequence alignment of $H B V$ and HIV-RT. Given the homology between HIV-RT and HBV polymerases, their primary amino acids sequences were aligned (Fig. 1A), corresponding positions were identified and mapped. As presented in Fig. 1A, in addition to YMDD which shared by HBV and HIV, positions 210-211 of HIV-RT and 228-229 of HBV-RT are conservative double leucines. HBV plasmids harboring different mutations of L228 and/or L229 were constructed by fusion PCR, and all mutations were located at the D domain of HBV-RT (Fig. 1B).

Within the HBV genome, the S gene overlaps with the $\mathrm{P}$ gene, meaning that one nucleotide substitution may alter the surface protein and the polymerase (19). In the present study, rtL228C/W resulted in $\mathrm{sF} 220 \mathrm{~V} / \mathrm{G}$, rtL229W gave rise to sC221G, while rtL228W/L229W led to sF220G/C221G (Table II). The HBV envelope proteins consist of small (S, $226 \mathrm{aa}$ ), middle (M, $281 \mathrm{aa}$ ), and large (L, $400 \mathrm{aa}$ ) proteins. $\mathrm{S}, \mathrm{M}$ and L, closely-associated transmembrane proteins, are in one single open reading frame (ORF) using three different in-frame translation start codons and a common stop codon.

The proposed topology of the HBV small envelope protein demonstrated that the small envelope protein traversed the endoplasmic reticulum (ER) membrane, probably four times $(20,21)$, between the $\mathrm{N}$ and $\mathrm{C}$ terminal (226 amino acids) (Fig. 1C), in order, there is the $\mathrm{N}$ terminal (residues 1 to 3 , exposed at the luminal side of the membrane), transmembrane domain I (TMD-I; between residues 4 and 28), a cytosolic loop between residues 28 and 80, TMD-II (between 100 and 164, containing the antigenic loop and a glycosylation site at Asn-146), TMD-III (between 173 and 193), TMD-IV (between 202 and 222) and the $\mathrm{C}$ terminal (between residues 223 and 226). As indicated in Fig. 1C, sF220 and sC221G were located at the $\mathrm{C}$-end of the protein.

Replication of pHBV1.3-rtXin transfected mammalian cells. Site substitutions in the $\mathrm{P}$ gene, including pHBV1.3-rtL228C,-rtL228W,-rtL229W and-rtL228W/L229W, were designed and constructed using fusion PCR to introduce into pBSK-HBV-WT plasmids. In order to further analyze the influence of these mutations on $\mathrm{HBV}$ replication, antigen production and sensitivity to NAs, together with pBSK-HBV1.3-wt, -rtM204V and -rtL180M/204V which were constructed in a
A

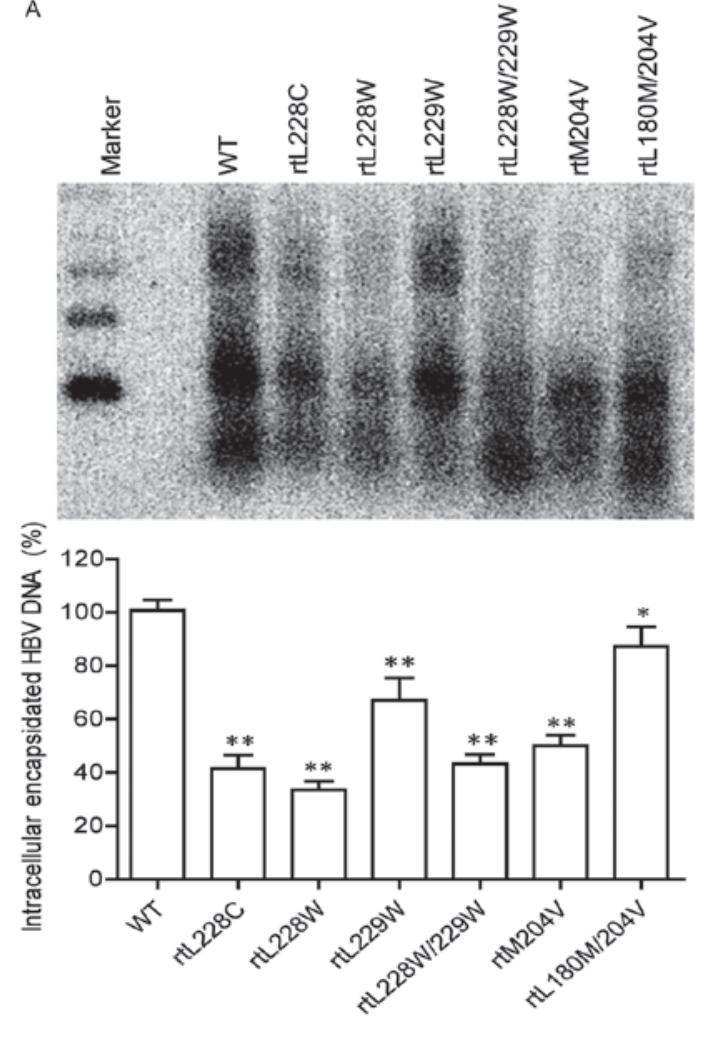

B
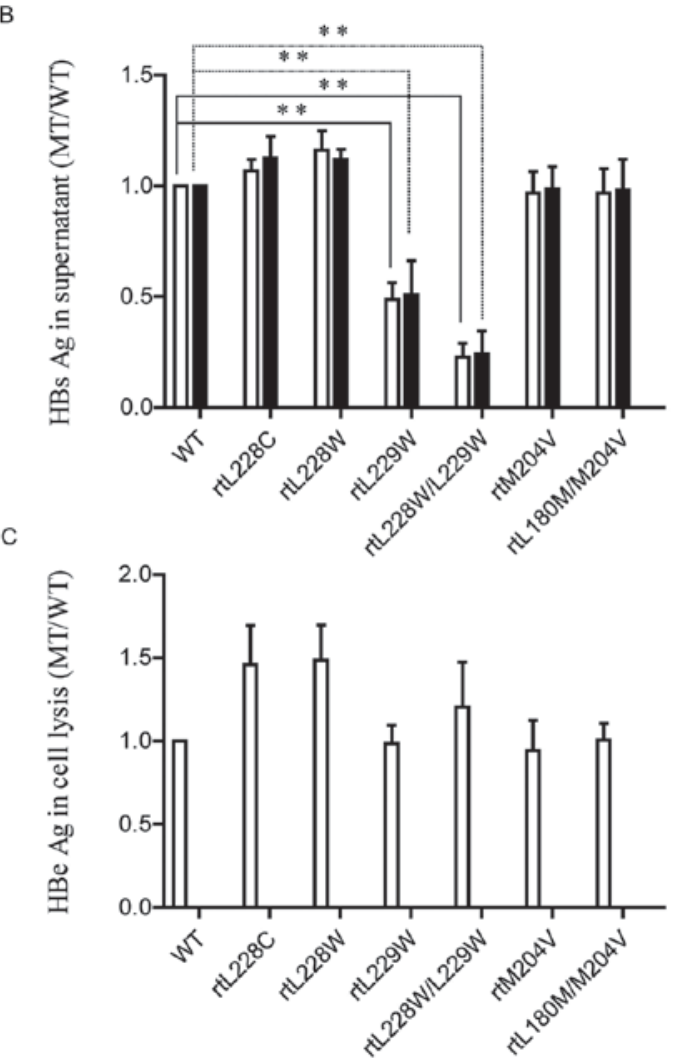

Figure 2. Huh7/HepG2 cells transfected with WT or MT HBV plasmids. (A) Encapsulated viral DNA was extracted and detected by Southern blotting. Relaxed circular, double stranded linear and single stranded HBV DNAs are illustrated. The relative level of WT HBV replication capacity was calculated as a percentage of the control via densitometric analysis. ${ }^{*} \mathrm{P}<0.05$; $^{* *} \mathrm{P}<0.01$ vs. WT. (B) HBsAg in the supernatant and cell lysate was detected using an HBsAg ELISA diagnostic kit, according to the manufacturer's protocol. ${ }^{* *} \mathrm{P}<0.01$. (C) HBeAg in the cell culture supernatant was tested using an $\mathrm{HBeAg}$ ELISA diagnostic kit. Each value is the mean of at least three independent experiments. The error bars represent the standard deviation. WT, wild-type; MT, mutant; HBV, hepatitis B virus; Ag, antigen. 

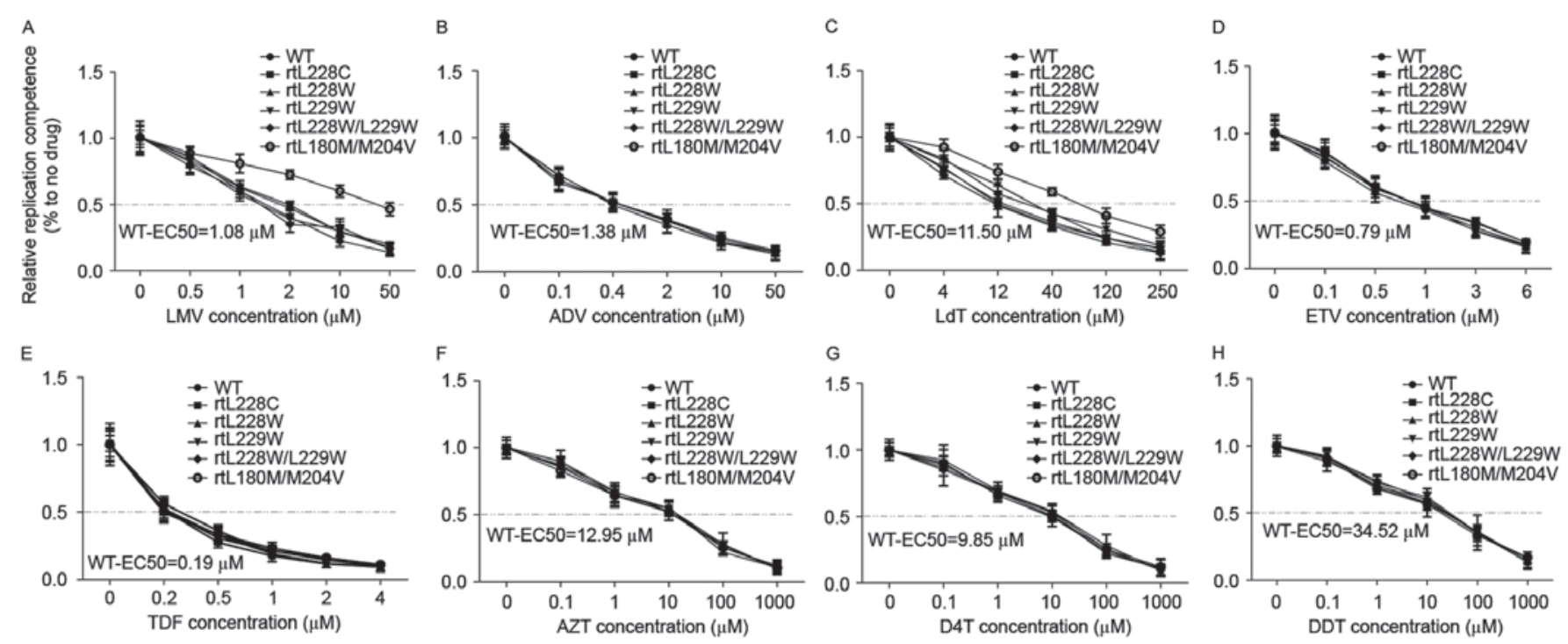

Figure 3. RT-qPCR detection of the anti-HBV effects of eight types of nucleos(t)ide analog. HepG2/Huh7 cells were transfected with the plasmids pHBV1.3-WT, -rtL228C, -rtL228W, -rtL229W, -rtL228W/L229W and -rtL180M/M204V, and subsequently treated with anti-HBV drugs at the indicated concentrations. Encapsulated HBV DNA was extracted and analyzed by RT-qPCR to evaluate the anti-HBV effects of (A) LMV, (B) ADV, (C) LdT, (D) ETV, (E) TDF, (F) AZT, (G) D4T and (H) DDI. RT-qPCR, reverse transcription-quantitative polymerase chain reaction; HBV, hepatitis B virus; LMV, lamivudine; ADV,

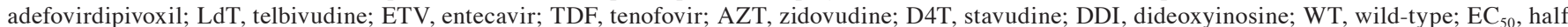
maximal effective concentration.

previous study (13), WT and MT HBV replication-competent plasmids were transfected into Huh7 cells and viral genome replication competence was analyzed by Southern blotting. Southern blotting and subsequent densitometric analysis (Fig. 2A) revealed that the substitutionsrt L228C, -rtL228W, -rtL229W and -rtL228W/L229W decreasedthe HBV replication capacity to $\sim 46,33,74$ and $52 \%$ of the WT, respectively. The mutation rtM204V/I was demonstrated to weaken HBV replication, while rtL180M was able to restore the impaired replication capacity, in accordance with a previous report (22).

HBsAg in the supernatant and cell lysate was detected using a commercial ELISA kit (Fig. 2B), as was HBeAg in the supernatant (Fig. 2C). The results indicated that none of the mutations led to a decrease in the supernatant HBeAg level compared with WT HBV. Compared with WT HBV, mutation $\mathrm{rtL} 228 \mathrm{C} / \mathrm{W}(\mathrm{sF} 220 \mathrm{~V} / \mathrm{G})$ enhanced the production and secretion of HBsAg, while rtL229W and rtL228W/L229W significantly decreased HBsAg expression in the supernatant and cell lysate. As the HBV polymerase gene is overlapped by the surface protein gene, mutations in polymerase may result in aa substitutions in HBsAg. In current study, rtM204V led to sI195M, but pBSK-HBV1.3-rtM204V and -rtL180M/204V exhibited an approximately consistent HBs/eAg level with WT HBV $(9,22)$. The results of the present study demonstrated that different substitutions resulted in different effects on HBV replication and HBsAg expression.

Inhibition of $H B V$ replication with NAs in vitro. In order to analyze the resistant phenotype of the different HBV mutations to NAs, the NA concentration was optimized usingp HBV1.3-WT in HepG2 cells. Following transfection with pHBV1.3-WT for $6 \mathrm{~h}$, different concentrations of the NAs were added into the fresh medium; core-associated HBV DNA in HepG2 cells was extracted and analyzed using the qPCR at $96 \mathrm{~h}(9)$. All the assayed drugs inhibited HBV DNA replication and the half maximal effective concentration values of 3TC, ADV, LdT, ETV, TDF, AZT, D4T and DDI were 1.08, 1.38, $11.60,0.79,0.19,12.95,9.85$ and $34.52 \mu \mathrm{M}$, respectively (Fig. 3). The results of the present study demonstrated that TDF was the most potent drug, followed by ETV, and that LdT, AZT, D4T and DDI exerted mild effects on HBV. Apart from pBSK-HBV1.3-rtM204V and -rtL180M/204V, which are classical LMV/LdT resistance mutations, the other MT HBV with comparable susceptibility were sensitive to the eight types of NA. In contrast to HIV, substitution mutations on L228 and/or L229 did not decreased the anti-HBV effect of the NAs. As reported previously, the nucleoside analogues did not affect the HBsAg, $\mathrm{HBeAg}$ and $\mathrm{HBcAg}$ expression (9).

\section{Discussion}

HBV replication is a complex dynamic process involving the interaction of a number of factors, comprising viruses, drugs and host factors (5). At present, thorough eradication of HBV in patients is not achievable using the available agents, including interferon and NAs (23). Certain NAs have been demonstrated to be effective anti-HBV agents in vivo and in vitro, although resistance mutations to NAs are selected during long-term anti-HBV therapy (6). Due to overlap between the polymerase gene and the surface protein gene, mutations in HBV polymerase may result in aa substitutions in HBsAg that potentially lead to immune evasion or modification of viral fitness. In a previous study, it was found that some new resistance mutations of HBV from the patients accepted anti-HBV therapy (6).

Previously, when a virological breakthrough occurred in a patient with chronic hepatitis B infection receiving one type of NA therapy, the HBV sequence was analyzed by sequencing and resistance mutations, if present, were detected. Not all resistance mutations were identified; certain mutations may be undetectable due to low frequency, and not all the detectable 
mutations exerted a marked effect due to error-prone reverse transcription and the phenomenon of quasispecies $(6,9)$. Additionally, this retrospective method is time-consuming and disadvantageous for clinical therapy. Therefore, prospective studies are required. Based on $3 \mathrm{D}$ modeling and the conservative sequence alignment of $\mathrm{HBV}$ and $\mathrm{HIV}$, certain resistance mutations may be predicted, as described in a previous study (9). Both rtL210 and rtL211 in HIV possess a double conservative leucine, according to the sequence alignment of HBV and HIV-RT, and the corresponding positions of HBV (rtL228 and rtL229) also possess a double conservative leucine. Furthermore, substitution rtL210W in $\mathrm{HIV}$ is an important resistance mutation (24). In the present study, in order to determine alterations in L228 and/or L229 of HBV, rtL228C, -rtL228W, -rtL229W and -rtL228W/L229W were designed and introduced into a WT HBV plasmid, termed pBSK-HBV1.3-WT (GenBank accession no. U95551), and LMV resistance mutations containing rtM204V and $\mathrm{rtL} 180 \mathrm{M} / 204 \mathrm{~V}$ were used as the control. Biomarkers of the viral life cycle and susceptibility to commonly used NAs were analyzed systematically. Due to the overlap between the polymerase gene and the surface protein gene of $\mathrm{HBV}$, mutations sF220V/G, sC221G and sF220G/C221G were driven by rtL228C/W, rtL229W and rtL228W/L229W, respectively. Amino acid sF220 and sC221 were located at TMD-IV, the carboxyl-terminal (between residues 164 and 226) of HBsAg, which has been reported to be highly hydrophobic and complex, and important for virion packaging (20). Cysteine is a natural amino acid containing sulfhydryl, which is able to form intra chain and inter chain disulfide bonds; these are important for the folding and stability of proteins, including in HBs (25). This may be the reason why rtL229W (sC221G) rtL228W/L229W (sF220G/C221G) significantly decrease HBsAg secretion. An altered sequence and structure of HBV-RT and HBs protein led by substitutions may directly influence viral replication capacity via alterations to the polymerase, or by indirect influences driven by HBV packaging through the interaction between surface and core protein of HBV, or a combination. In the present study, single or amphimutationsinrt L228 and rtL229 significantly impaired HBV replication. The detailed mechanism underlying this observation requires further study.

The HepG2 cell line used in the present study is thought to be derived from hepatoblastoma rather than hepatocarcinoma (26). HepG2 and Huh7 cell lines were used in present study. With regard to the replication capacity, the HBs/e antigen level and the susceptibility to NAs of the WT and MT HBV, similar conclusions were drawn from HepG2 and Huh7; therefore, only the results of HepG2 are presented, and those of Huh7 are not shown. In a previous study, HBs/eAg levels in the supernatant from Huh7 or HepG2 transfected with HBV plasmids were not consistent with the level of HBV replication. Notably, in transfected systems, NAs selectively inhibit HBV DNA and not HBs/eAg (9). However, specific small interfering RNA may inhibit HBV DNA and HBs/eAg in the same system (27). In the present study, rtL229W and rtL228W/L229W inhibited HBsAg production due to point mutations in the surface protein. Different from HBsAg, for $\mathrm{HBeAg}$ in supernatant, due to the intact polypeptide sequence of the $\mathrm{HBe}$ antigen, there was no significant difference between WT and MT HBV. These results were consistent previous research (9). However, the results of the resistance phenotype analysis were not in line with previous findings. Mutations in L228 and/or L229 did not decrease the anti-HBV effect of NAs, while in the same experimental system, rtM204V and rtL180M/204V were resistant to LMV and LdT compared with WT HBV. Despite the similar RT structure shared by HBV and HIV, one aa alteration may exert different influences on the RT of HBV and HIV. The results of the present study demonstrated that $2 \mathrm{D}$ sequence alignment alone is insufficient for the prediction of resistance mutations, and requires the addition of 3D spatial structure analysis.

\section{Acknowledgements}

The present study was supported by the Medical Scientific Research Foundation of Zhejiang Province, China (grant no. 2013KYA208), and the Shaoxing Science and Technology Bureau, Zhejiang Province of China (grant no. 2013B70063).

\section{References}

1. Li W and Urban S: Entry of hepatitis B and hepatitis D virus into hepatocytes: Basic insights and clinical implications. J Hepatol 64 (1 Suppl): S32-S40, 2016.

2. Zhang Q, Liao Y, Chen J, Cai B, Su Z, Ying B, Lu X, Tao C and Wang L: Epidemiology study of HBV genotypes and antiviral drug resistance in multi-ethnic regions from Western China. Sci Rep 5: 17413, 2015.

3. Bartholomeusz A, Tehan BG and Chalmers DK: Comparisons of the HBV and HIV polymerase and antiviral resistance mutations. Antivir Ther 9: 149-160, 2004.

4. Dandri M,Lutgehetmann M and Petersen J: Experimental models and therapeutic approaches for HBV. Semin Immunopathol 35: 7-21, 2013 .

5. Zoulim F and Durantel D: Antiviral therapies and prospects for a cure of chronic hepatitis B. Cold Spring Harb Perspect Med 5: a021501, 2015.

6. Zoulim F and Locarnini S: Hepatitis B virus resistance to nucleos(t)ide analogues. Gastroenterology 137: 1593-1608.e1-2, 2009.

7. Cabuang LM, Shaw T, Littlejohn M, Colledge D, Sozzi V, Soppe S, Warner N, Thompson A, Preiss S, Lam N, et al: In vitro replication phenotype of a novel $(-1 \mathrm{G})$ hepatitis B virus variant associated with HIV co-infection. J Med Virol 84: 1166-1176, 2012.

8. Petersen J, Thompson AJ and Levrero M: Aiming for cure in HBV and HDV infection. J Hepatol 65: 835-848, 2016.

9. Qin B, Budeus B, Cao L, Wu C, Wang Y, Zhang X, Rayner S, Hoffmann D, Lu M and Chen X: The amino acid substitutions $\mathrm{rtP} 177 \mathrm{G}$ and $\mathrm{rtF} 249 \mathrm{~A}$ in the reverse transcriptase domain of hepatitis B virus polymerase reduce the susceptibility to tenofovir. Antiviral Res 97: 93-100, 2013.

10. Aragri M, Alteri C, Battisti A, Di Carlo D, Minichini C, Sagnelli C, Bellocchi MC, Pisaturo MA, Starace M, Armenia D, et al: Multiple Hepatitis B virus (HBV) quasispecies and immune-escape mutations are present in HBV surface antigen and reverse transcriptase of patients with acute hepatitis B. J Infect Dis 213: 1897-1905, 2016.

11. Caviglia GP, Abate ML, Noviello D, et al: Hepatitis B core-related antigen kinetics in chronic HBV-genotype-D infected patients treated with nucleos(t)ide analogues or pegylated-interferon-alpha. Hepatol Res, 2016

12. Kim HS, Yim HJ, Jang MK, Park JW, Suh SJ, Seo YS, Kim JH, Kim BH, Park SJ, Lee SH, et al: Management of entecavirresistant chronic hepatitis $\mathrm{B}$ with adefovir-based combination therapies. World J Gastroenterol 21: 10874-10882, 2015.

13. Qin B,Zhang B,Zhang X,He T, Xu W, Fu L and Tu C: Substitution $\mathrm{rtq} 267 \mathrm{~h}$ of hepatitis $\mathrm{B}$ virus increases the weight of replication and lamivudine resistance. Hepat Mon 13: e12160, 2013.

14. Yahi N, Tamalet C, Tourres C, Tivoli N and Fantini J: Mutation L210W of HIV-1 reverse transcriptase in patients receiving combination therapy. Incidence, association with other mutations and effects on the structure of mutated reverse transcriptase. J Biomed Sci 7: 507-513, 2000 . 
15. Clavel F and Hance AJ: HIV drug resistance. N Engl J Med 350: 1023-1035, 2004.

16. Delviks-Frankenberry KA, LengruberRB, Santos AF, Silveira JM, Soares MA, Kearney MF, MaldarelliF and Pathak VK: Connection subdomain mutations in HIV-1 subtype-C treatment-experienced patients enhance NRTI and NNRTI drug resistance. Virology 435: 433-441, 2013.

17. Qin B, He T, Chen Z, Xu W, Pan G and Tu C: A novel method for the analysis of drug-resistant phenotypes of hepatitis B virus. Int J Mol Med 31: 975-981, 2013.

18. Pokrovsky AG, Pronayeva TR, Fedyuk NV, Shirokova EA, Khandazhinskaya AL, Tarusova NB, Karpenko IL and Krayevsky AA: Anti-HIV activity of novel phosphonate derivatives of AZT, d4T and ddA. Nucleosides, Nucleotides Nucleic Acids 20: 767-769, 2001.

19. Su M, Xiang K, Li Y, Li Y, Deng J, Xu X, Yan L, Zhuang H and Li T: Higher detection rates of amino acid substitutions in HBV reverse transcriptase/surface protein overlapping sequence is correlated with lower serum HBV DNA and HBsAg levels in $\mathrm{HBeAg}$-positive chronic hepatitis B patients with subgenotype B2. Infect Genet Evol 40: 275-281, 2016.

20. Khan N, Guarnieri M, Ahn SH, Li J, Zhou Y, Bang G, Kim KH, Wands JR and Tong S: Modulation of hepatitis B virus secretion by naturally occurring mutations in the $\mathrm{S}$ gene. J Virol 78 : 3262-3270, 2004.

21. Abou-Jaoudé G, Molina S, Maurel P and Sureau C: Myristoylation signal transfer from the large to the middle or the small HBV envelope protein leads to a loss of HDV particles infectivity. Virology 365: 204-209, 2007.
22. Li MW, Hou W, Wo JE and Liu KZ: Character of HBV (hepatitis B virus) polymerase gene rtM204V/I and rtL180M mutation in patients with lamivudine resistance. J Zhejiang Univ Sci B 6: 664-667, 2005.

23. Lucifora J, Xia Y, Reisinger F, Zhang K, Stadler D, Cheng X, Sprinzl MF, Koppensteiner H, Makowska Z, Volz T, et al: Specific and nonhepatotoxic degradation of nuclear hepatitis B virus cccDNA. Science 343: 1221-1228, 2014

24. Fourati S, Malet I, Guenzel CA, Soulie C, Maidou-Peindara P, Morand-Joubert L, Wirden M, Sayon S, Peytavin G, Simon A, et al: E17A mutation in HIV-1 Vpr confers resistance to didanosine in association with thymidine analog mutations. Antiviral Res 93: 167-174, 2012.

25. Michel M, Lone YC, Centlivre M, Roux P, Wain-Hobson S and Sala M: Optimisation of secretion of recombinant HBsAg virus-like particles: Impact on the development of HIV-1/HBV bivalent vaccines. Vaccine 25: 1901-1911, 2007.

26. Lopez-Terrada D, Cheung SW, Finegold MJ and Knowles BB: Hep G2 is a hepatoblastoma-derived cell line. Hum Pathol 40: 1512-1515, 2009.

27. Pei R, Qin B, Zhang X, Zhu W, Kemper T, Ma Z, Trippler M, Schlaak J, Chen X and Lu M: Interferon-induced proteins with tetratricopeptide repeats 1 and 2 are cellular factors that limit hepatitis B virus replication. J Innate Immun 6: 182-191, 2014. 